\title{
Quasinormal modes of the near extremal Schwarzschild-de Sitter black hole
}

\author{
Vitor Cardosd $*$ and José P. S. Lemođt \\ Centro Multidisciplinar de Astrofísica - CENTRA, \\ Departamento de Física, Instituto Superior Técnico, \\ Av. Rovisco Pais 1, 1049-001 Lisboa, Portugal
}

(Dated: October 23, 2018)

\begin{abstract}
We present an exact expression for the quasinormal modes of scalar, electromagnetic and gravitational perturbations of a near extremal Scwarzschild-de Sitter black hole and we show why a previous approximation holds exactly in this near extremal regime. In particular, our results give the asymptotic behavior of the quasinormal frequencies for highly damped modes, which has recently attracted much attention due to the proposed identification of its real part with the Barbero-Immirzi parameter.

PACS numbers: 04.70.Bw, 04.70.-s, 04.30.-w
\end{abstract}

\section{INTRODUCTION}

The quasinormal modes of spacetimes containing black holes have proved to be extremely important in several astrophysical aspects; for instance, they allow us to ascertain whether or not the spacetime is stable against deviations from equilibrium, and they give us invaluable information on what kind of signal one expects if one perturbs the spacetime. In fact the quasinormal modes and their associated frequencies are a signature of the spacetime, in that they depend only on the conserved charges, such as the mass and electrical charge, making it possible to identify the spacetime just by seeking its quasinormal frequencies. This has motivated a wide effort to find the quasinormal frequencies, and several numerical and analytical techniques have been devised 1].

It has been also realized that the quasinormal modes are important in the context of the AdS/CFT conjecture [2, 3]. According to it, the black hole corresponds to a thermal state in the conformal field theory, and the decay of the test field in the black hole spacetime corresponds to the decay of the perturbed state in the CFT. The dynamical timescale for the return to thermal equilibrium is very hard to compute directly, but can be done relatively easily using the AdS/CFT correspondence. This has motivated a search for the quasinormal modes in asymptotically anti-de Sitter black holes [3, 4, 5, 6].

Very recently, the quasinormal modes have acquired a different importance. Following an observation by Hod [7], it has been proposed [8] that the Barbero-Immirzi parameter 9], a factor introduced by hand in order that Loop Quantum Gravity reproduces correctly the black hole entropy, is equal to the real part of the quasinormal frequencies with a large imaginary part. The identification came from what first seemed to be a numeri-

\footnotetext{
*Electronic address: vcardoso@fisica.ist.utl.pt
}

${ }^{\dagger}$ Electronic address: lemos@kelvin.ist.utl.pt cal coincidence, but which has been proved to be exact for Schwarzschild black holes by Motl [10], assuming the gauge group of the theory to be $S O(3)$.

It is now important to see whether the agreement works only for Schwarzschild black holes, or if it continues to be true in different spacetimes, for example higher dimensional Schwarzschild spacetime (where some of the lowest lying scalar quasinormal frequencies have already been computed 11]), or Schwarzschild-de Sitter or antide Sitter spacetimes. In this work we shall take a step further on carrying on this program by computing exactly the quasinormal frequencies of the near extremal Schwarzschild-de Sitter black hole, which is the spacetime for which the black hole horizon and the cosmological horizon are close to each other, in a manner to be defined latter. For this spacetime, we find that it is possible to solve the field equations exactly in terms of hypergeometric functions, and therefore an exact analytical expression for the quasinormal frequencies of scalar, electromagnetic and gravitational perturbations is also possible. In particular this will give us the quasinormal frequencies with very large imaginary part. We demonstrate why an approach by Moss and Norman [5] based on fitting the potential to the Pöshl-Teller potential works well in the Schwarzschild-de Sitter spacetime.

\section{EQUATIONS}

Our notation will follow that of [12] which we have found convenient. The metric of the Schwarzschild-de Sitter (SdS) spacetime is given by

$$
d s^{2}=-f d t^{2}+f^{-1} d r^{2}+r^{2}\left(d \theta^{2}+\sin ^{2} \theta d \phi^{2}\right),
$$

where

$$
f=1-\frac{2 M}{r}-\frac{r^{2}}{a^{2}},
$$

with $M$ denoting the black-hole mass, and $a^{2}$ is given in terms of the cosmological constant $\Lambda$ by $a^{2}=3 / \Lambda$. The 
spacetime possesses two horizons: the black-hole horizon is at $r=r_{b}$ and the cosmological horizon is at $r=r_{c}$, where $r_{c}>r_{b}$. The function $f$ has zeroes at $r_{b}, r_{c}$, and $r_{0}=-\left(r_{b}+r_{c}\right)$. In terms of these quantities, $f$ can be expressed as

$$
f=\frac{1}{a^{2} r}\left(r-r_{b}\right)\left(r_{c}-r\right)\left(r-r_{0}\right)
$$

It is useful to regard $r_{b}$ and $r_{c}$ as the two fundamental parameters of the SdS spacetime, and to express $M$ and $a^{2}$ as functions of these variables. The appropriate relations are

$$
a^{2}=r_{b}^{2}+r_{b} r_{c}+r_{c}^{2}
$$

and

$$
2 M a^{2}=r_{b} r_{c}\left(r_{b}+r_{c}\right) .
$$

We also introduce the surface gravity $\kappa_{b}$ associated with the black hole horizon $r=r_{b}$, as defined by the relation $\kappa_{b}=\frac{1}{2} d f / d r_{r=r_{b}}$. Explicitly, we have

$$
\kappa_{b}=\frac{\left(r_{c}-r_{b}\right)\left(r_{b}-r_{0}\right)}{2 a^{2} r_{b}} .
$$

After a Fourier decomposition in frequencies and a multipole expansion, the scalar, electromagnetic and gravitational perturbations all obey a wave equation of the form $[4,6]$

$$
\frac{\partial^{2} \phi(\omega, r)}{\partial r_{*}^{2}}+\left[\omega^{2}-V(r)\right] \phi(\omega, r)=0,
$$

where the tortoise coordinate is given by

$$
r_{*} \equiv \int f^{-1} d r
$$

and the potential $V$ depends on the kind of field under consideration. Explicitly, for scalar perturbations

$$
V_{\mathrm{s}}=f\left[\frac{l(l+1)}{r^{2}}+\frac{2 M}{r^{3}}-\frac{2}{a^{2}}\right],
$$

while for electromagnetic perturbations

$$
V_{\mathrm{el}}=f\left[\frac{l(l+1)}{r^{2}}\right] .
$$

The gravitational perturbations decompose into two sets [4], the odd and the even parity one. We find however that for this spacetime, they both yield the same quasinormal frequencies, so it is enough to consider one of them, the odd parity ones say, for which the potential is 4]

$$
V_{\text {grav }}=f\left[\frac{l(l+1)}{r^{2}}-\frac{6 M}{r^{3}}\right] .
$$

In all cases, we denote by $l$ the angular quantum number, that gives the multipolarity of the field.

\section{A. The Near Extremal SdS Black Hole}

Let us now specialize to the near extremal SdS black hole, which is defined as the spacetime for which the cosmological horizon $r_{c}$ is very close (in the $r$ coordinate) to the black hole horizon $r_{b}$, i.e. $\frac{r_{c}-r_{b}}{r_{b}}<<1$. For this spacetime one can make the following approximations

$$
r_{0} \sim-2 r_{b}^{2} ; a^{2} \sim 3 r_{b}^{2} ; M \sim \frac{r_{b}}{3} ; \kappa_{b} \sim \frac{r_{c}-r_{b}}{2 r_{b}^{2}} .
$$

Furthermore, and this is the key point, since $r$ is constrained to vary between $r_{b}$ and $r_{c}$, we get $r-r_{0} \sim$ $r_{b}-r_{0} \sim 3 r_{0}$ and thus

$$
f \sim \frac{\left(r-r_{b}\right)\left(r_{c}-r\right)}{r_{b}^{2}} .
$$

In this limit, one can invert the relation $r_{*}(r)$ of (8) to get

$$
r=\frac{r_{c} e^{2 \kappa_{b} r_{*}}+r_{b}}{1+e^{2 \kappa_{b} r_{*}}} .
$$

Substituting this on the expression (13) for $f$ we find

$$
f=\frac{\left(r_{c}-r_{b}\right)^{2}}{4 r_{b}^{2} \cosh \left(\kappa_{b} r_{*}\right)^{2}} .
$$

As such, and taking into account the functional form of the potentials (91)-(11) we see that for the near extremal SdS black hole the wave equation (7) is of the form

$$
\frac{\partial^{2} \phi(\omega, r)}{\partial r_{*}^{2}}+\left[\omega^{2}-\frac{V_{0}}{\cosh \left(\kappa_{b} r_{*}\right)^{2}}\right] \phi(\omega, r)=0,
$$

with

$$
V_{0}= \begin{cases}\kappa_{b}^{2} l(l+1), & \text { scalar and electromagnetic } \\ \kappa_{b}^{2}(l+2)(l-1), & \text { perturbations. } \\ & \text { pravitational } \\ & \text { perturbations }\end{cases}
$$

The potential in (16) is the well known Pöshl-Teller potential [13]. The solutions to (16) were studied and they are of the hypergeometric type, (for details see Ferrari and Mashhoon 14]). It should be solved under appropriate boundary conditions:

$$
\begin{array}{r}
\phi \sim e^{-i \omega r_{*}}, r_{*} \rightarrow-\infty \\
\phi \sim e^{i \omega r_{*}}, r_{*} \rightarrow \infty .
\end{array}
$$

These boundary conditions impose a non-trivial condition on $\omega$ [14], and those that satisfy both simultaneously are called quasinormal frequencies. For the Pöshl-Teller potential one can show [14] that they are given by

$$
\omega=\kappa_{b}\left[-\left(n+\frac{1}{2}\right) i+\sqrt{\frac{V_{0}}{\kappa_{b}^{2}}-\frac{1}{4}}\right], n=0,1, \ldots .
$$


Thus, with (17) one has

$$
\frac{\omega}{\kappa_{b}}=-\left(n+\frac{1}{2}\right) i+\sqrt{l(l+1)-\frac{1}{4}}, n=0,1, \ldots .
$$

for scalar and electromagnetic perturbations. And

$$
\frac{\omega}{\kappa_{b}}=-\left(n+\frac{1}{2}\right) i+\sqrt{(l+2)(l-1)-\frac{1}{4}}, n=0,1, \ldots .
$$

for gravitational perturbations. Our analysis shows that Eqs. (21)-22) are correct up to terms of order $O\left(r_{c}-r_{b}\right)$ or higher. Moss and Norman [5] have studied the quasinormal frequencies in the SdS geometry numerically and also analytically, by fitting the potential to a Pöshl-Teller potential. Their analytical results (see their Figs 1 and 2) were in excellent agreement with their numerical results, and this agreement was even more remarkable for near extremal black holes and for high values of the angular quantum number $l$. We can now understand why: for near extremal black holes the true potential is indeed given by the Pöshl-Teller potential! Furthermore for near extremal SdS black holes and for high $l$ our formula (22) is approximately equal to formula (19) of Moss and Norman [5]. With their analytical method of fitting the potential one can never be sure if the results obtained will continue to be good as one increases the mode number $n$. But we have now proved that if one is in the near extremal SdS black hole, the Pöshl-Teller is the true potential, and so Eq. (21)-(22) is exact. For example, Moss and Norman obtain numerically, and for gravitational perturbations with $l=2$ of nearly extreme SdS black holes, the result

$$
\frac{\omega_{\mathrm{num}}}{\kappa_{b}}=1.93648-i\left(n+\frac{1}{2}\right),
$$

and we obtain, from (22)

$$
\frac{\omega}{\kappa_{b}}=1.936492-i\left(n+\frac{1}{2}\right) .
$$

For $l=3$ Moss and Norman [5] obtain

$$
\frac{\omega_{\text {num }}}{\kappa_{b}}=3.12249-i\left(n+\frac{1}{2}\right),
$$

and we obtain, from (22)

$$
\frac{\omega}{\kappa_{b}}=3.122499-i\left(n+\frac{1}{2}\right) .
$$

So this remarkable agreement allows us to be sure that (21)-(22) are indeed correct.

\section{CONCLUSIONS}

We have found an analytical expression for the quasinormal modes and frequencies of a nearly extreme schwarzschild-de Sitter black hole. This expression, Eqs. (21)-(22) are correct up to terms of order $O\left(r_{c}-r_{b}\right)$ or higher for all $n$. This means that we can be confident that for high overtones, i.e. large $n$, our expression is still valid. One can see that the real part of the quasinormal frequency does not depend on the integer $n$ labelling the mode. Therefore, frequencies with a large imaginary part still have a real part given by $\sqrt{l(l+1)-\frac{1}{4}}$ for scalar and electromagnetic perturbations and by $\sqrt{(l+2)(l-1)-\frac{1}{4}}$ for gravitational perturbations. Can one explain an highly damped quasinormal frequency with an $l$-dependent real part in light of the recent conjectures [8] relating it to the Barbero-Immirzi parameter? We think it is too early to answer this, and much more work is still necessary, specially in higher dimensional spacetimes, and on Anti-de Sitter spacetimes [15], where the AdS/CFT conjecture may have a word to say about this.

\section{Acknowledgements}

It is a pleasure to acknowledge stimulating conversations with Óscar Dias and Lubos Motl. We are specially grateful to Ian Moss and James Norman, who kindly provided their numerical results. This work was partially funded by Fundação para a Ciência e Tecnologia (FCT) Portugal through project PESO/PRO/2000/4014. V.C. also acknowledges finantial support from FCT through PRAXIS XXI programme. J. P. S. L. thanks Observatório Nacional do Rio de Janeiro for hospitality.
[1] K. D. Kokkotas and B. G. Schmidt, Living Rev. Rel. 2, 2(1999).

[2] J. Maldacena, Adv. Theor. Math. Phys. 2, 253(1998).

[3] G. T. Horowitz and V. E. Hubeny Phys. Rev. D62, 024027 (2000).

[4] V. Cardoso and J. P. S. Lemos, Phys. Rev. D64, 084017
(2001);

[5] I. G. Moss and J. P. Norman, Class. Quant. Grav. 19, 2323-2332 (2002).

[6] V. Cardoso and J. P. S. Lemos, Phys. Rev. D63, 124015 (2001); Class. Quant. Grav. 18, 5257 (2001); R. A. Konoplya, Phys. Rev. D66, 044009 (2002); D. Birmingham, 
I. Sachs and S. N. Solodukhin, Phys. Rev. Lett. 88, 151301 (2002); hep-th/0212308 S. F. J. Chan and R. B. Mann, Phys. Rev. D55, 7546 (1997); R. Aros, C. Martinez, R. Troncoso and J. Zanelli, hep-th/0211024 D. T. Son, A. O. Starinets, J.H.E.P. 0209:042, (2002); A. O. Starinets, hep-th/0207133 W. T. Kim and J. J. Oh, Phys. Lett. B514, 155 (2001); N. Iizuka, D. Kabat, G. Lifschytz and D. A. Lowe, hep-th/0212246 Y. Kurita and M. Sakagami, hep-th/0208063 S. Musiri and G. Siopsis, hep-th/0301081 E. Berti and K.D. Kokkotas, gr-qc/0301052

[7] S. Hod, Phys. Rev. Lett. 81, 4293 (1998).

[8] O. Dreyer, gr-qc/0211076 G. Kunstatter, gr-qc/0211076 A. Corichi, gr-qc/0212126
[9] G. Immirzi, Nucl. Phys. Proc. Suppl. 57, 65 (1997).

[10] L. Motl, gr-qc/0212096

[11] V. Cardoso, Óscar J. C. Dias and J. P. S. Lemos, hep-th/0212168

[12] P. Brady, C. Chambers, W. Laarakkers and Eric Poisson, Phys. Rev. D60, 064003 (1999).

[13] G. Pöshl and E. Teller, Z. Phys. 83, 143 (1933).

[14] V. Ferrari and B. Mashhoon, Phys. Rev. D30, 295 (1984).

[15] V. Cardoso, R. Konoplya and J. P. S. Lemos, Asymptotic behavior of the quasinormal frequencies of Schwarzschildanti-de Sitter spacetimes, in preparation. 